\title{
A Semi-Empirical Model of the Wheel-Soil System
}

\author{
Piotr Tarkowski ${ }^{*}$
}

1 Department of Automotive Vehicles, Faculty of Mechanical Engineering, Lublin University of Technology, Nadbystrzycka 36, 20-618 Lublin, Poland

e-mail: p.tarkowski@pollub.pl

\begin{abstract}
The article presents a proposal of a semi-empirical model of a wheel - soil system. Measurements of the values characterizing this cooperation were carried out with the use of a measuring and test stand, consisting of the SST sensor for measuring the soil stress components and a dynamometer for measuring forces acting on the road wheel. The research was carried out on three different soils: sandy, loess and peat. Graphs showing the correlation between forces and stresses in the substrate were obtained. It was observed the influence of soil type and other parameters on the type and course of the curves in these graphs.
\end{abstract}

Keywords: rolling resistance, wheel-soil interaction, deformable ground.

\section{THE MOTIVATION}

The analysis of the system vehicle wheel - deformable ground is a dynamic and spatial issue. Especially the deformability of the ground (volumetric and form) has an impact on the driving properties and the performance of the vehicle, as it causes an rolling resistance [1].

Problems related to the description of the wheel-soil system existed before the invention of motor vehicles. Deformation of the soil may be of a plastic character. The theoretical foundations for solving this problem were created by Coulomb. $\mathrm{He}$ proposed the so-called the two-variable hypothesis:

$$
\tau=C+p \operatorname{tg}(\varphi)
$$

Where: $\tau$-tangential stress, $C$ - cohesion, $p-$ normal stress, $\varphi-$ angle of internal friction.

Using the formula (1), it is possible to determine the value of the tangential (shear) stress on the contact surface of the wheel with the ground, on which the normal stress $p$ prevails, and the soil material is characterized by the cohesion $C$ and the internal friction angle $\varphi$. The so-called two constants hypothesis turned out to be useful in solving terramechanical problems. Today, many models of wheel-soil interaction are based on this fundamental theory [2]. However, the Coulomb hypothesis does not provide any information on the deformation of the soil material. Considering the high deformability of subsoils, it can be a source of serious errors.

In the 1960s, the discipline of terramechanics developed. The creator of it is considered to be G.M. Bekker [3]. He conducted research in the field of cooperation of the running components with various deformable grounds. The result of these studies is a semi-experimental model in which the soil is described using the $K$ coefficient, characterizing its deformability and the so-called dimensionless soil state exponent $n$ :

$$
p=K z^{n}
$$

Where: $p$ - normal stress, $z$ - rut depth.

This model, along with modifications, has application in many scientific studies, as well as in practice, for example as a tool for improving the design of elements and running gears of vehicles. In conjunction with the Coulomb model, the Bekker formula allows the determination of traction forces: rolling resistance force and driving force - especially for tracked undercarriages on sandy 
grounds. In the case of circular track systems, the Bekker formula gives less accurate results. Loess and clay soils are characterized by the presence of a peak on the shear curve, which results in imprecise determination of the driving force [4-7].

The inconvenience of simple calculation methods prompted the search for solutions with wider possibilities. Especially high-deformation grounds, such as loose soil or fresh snow, and pneumatic tires, require the development of new test methods.

The main task is to describe the mechanics of the soil deformation process. Because the socalled constitutive equations (the relationships between stress and strains for materials such as soil) are not known, a good way is to do experimental tests and attempts to describe the layout of the wheel - soil based on empirical models.

Initially, the main aim of the research was to determine the adverse effect of soil loading, the so-called soil compaction. These types of models were based on the relationship between the stress state and the resulting density of compacted soil. The sensors and measuring equipment used did not allow sampling the stress state with high frequency under dynamic conditions. Systems based on microprocessor systems and fast transducers adapted to dynamic measurement conditions made it possible to measure the stress during vehicle maneuvers [8-11].

\section{General assumptions for the model of the wheel - soil system}

Modeling the wheel-soil system is possible in four ways: (1) on the basis on experimental data only, (2) using similarity methods, (3) using simple physical models, (4) as complex physical models.

The first group includes models based on the experimental results obtained in studies with real objects in full scale. These types of models use regression techniques to fit the waveforms of the measured values and mathematical relationships. The next groups of models are based more on the theoretical foundations of tyre behavior, while the most complex models of the group (4) may give the most accurate results of the simulation, but due to their complexity, the necessary effort and time needed to perform numerical calculations, their usefulness in tests vehicle dynamics is limited. Models from group (1) are characterized by a certain structure, described by parameters that can be obtained through tests with the use of real objects. An example is the
MAGIC FORMULA model, in which there are formulas based on trigonometric functions that give a very good fit to the courses of the real forces $F_{y}, F_{x}$ and the moment $M_{z}[12]$.

Pneumatic tyre - soft soil ground system can be described by the forces acting on the wheel and the stress generated in the ground. From the point of view of mechanics, the interaction of a wheel is an action, and the development of stress in the ground is a reaction. There are flexible bodies along the way between the wheel axis and the stress measurement point, which makes this relationship complicated. First of all, the deformability of the substrate as well as the deformability of the tire affect the relationship between forces and stress. The tyres and soil form a system that partially transfers loads and partially absorbs them. The nature of the force-stress relationship depends on the generally understood deformability of both elements. In practice, it is difficult to characterize tyres and ground using one parameter, the more so as it is a rather theoretical parameter.

A typical analytical model should take into account these factors in the form of parameters or even functions. Considering that only the most important factors are listed here - and in general it can be expected that the final form of the model equations would be extremely complicated, while the parameterization of such a model would be a serious difficulty.

For the above reasons, the main purpose of the work was to develop a model of the tyre wheel - deformable ground system using the identification method, in which the input and output values describing a system are known, and as a result of numerical analysis of in/out signals mathematical models are obtained, that take into account the above-mentioned factors.

\section{Model identification experiment}

One of the conditions for the identification of the model is to design the experiment in such a way, that the obtained results will constitute input and output signals uniquely characterizing the subject system. Therefore, it is necessary to plan the possibility of measuring all input quantities forces acting on the half-space of the soil medium and outputs, soil stresses. Hence the initial requirements as to the necessary test methodology and measuring system. 


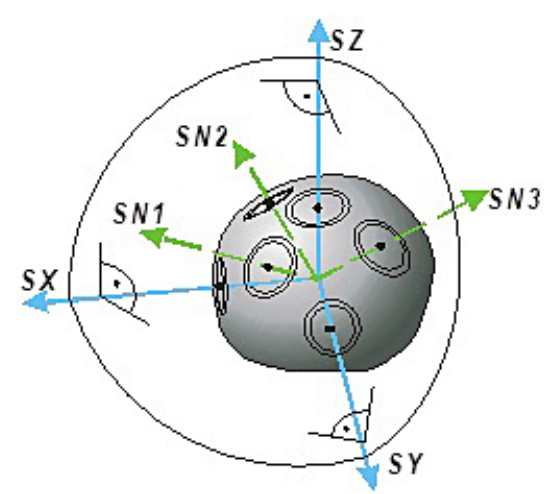

Fig. 1. Diagram of the SST sensor for measuring the soil stress state components

\section{Measurement of wheel forces}

A method of continuous measurement of the forces acting on vehicle wheel is necessary. Three forces must be measured simultaneously: vertical, longitudinal and transverse. The sampling frequency was assumed at the level of $100 \mathrm{~Hz}$ for each measurement channel. The tests used a rotating wheel dynamometer embedded in the wheel. The dynamometer measures 6 components: three forces acting in the direction of the main axes and the corresponding moments [13-16].

\section{Measurement of soil stress}

SST sensors, which are six-transducers sensors, capable of measurement of the stress components, were used to measure the soil stress state [17-21]. The stresses in soil were measured with the same frequency as for force measurements. The SST sensor enabled direct measurement of the axial components of the stress tensor $\sigma_{z}, \sigma_{x}, \sigma_{y}$ and the determination of the tangent components.

\section{RESULTS}

\section{Correlations of wheel forces and soil stresses}

The first stage of the research was an attempt to obtain the simplest model, based on data from measurements in real conditions. As it was assumed that the research was to be carried out in the field of longitudinal and vertical dynamics of the wheel - soil system, hence the selection of the values of forces and stresses. Based on the measurement results, the following correlations of the forces with the relevant components of the stress state in the soil were made:
- vertical (load) force $F_{z}$ and vertical stress $\sigma_{z}$;

- horizontal (driving) force $F_{x}$ and horizontal stress $\sigma_{x}$.

These correlations describe the most important mutual interactions in the wheel - soil system. In order to obtain a correlation, a fragment of the force course and the corresponding fragment of the stress course were analyzed. Then, the range of force values for which the stress (strain) reached values greater than the rest was distinguished. Good correlation of the components of the stress state and forces required that certain characteristic points in the graphs of both these quantities were consistent in time. The waveforms were prepared in such a way, that for half of the full cycle measured by the wheel dynamometer, an equal length course of the appropriate stress was selected, with the peak of this waveform set in the middle of the range, so that it coincides with the peak (or zero) of the force on the wheel. Such "matching" of the values of forces waveforms and stress components does not significantly affect the final result, because the phase shift in relation to the position of the actual ranges of values is insignificant. For example, if we shift the separated range of stress components by approx. $100 \mathrm{~ms}$ (10 time units on the scale), then at a speed of 5-7 km/h during measurements, it gives a total displacement of $200 \mathrm{~mm}$. This corresponds to a situation where the force measurement would be shifted from the stress measurement by the above-mentioned 200 $\mathrm{mm}$. Safely, it can be assumed that the continuity of the soil ensures, that the conditions within these $200 \mathrm{~mm}$ do not change enough to significantly affect the measurement results.

Exemplary waveforms of forces values and stress components are shown in Figure 2. The waveforms listed there are not consistent on the scale, and the purpose of the drawing is to present the shape and mutual location of the waveforms measured simultaneously. It is clear that the forces $F_{x}$ and $F_{z}$ are mutually shifted in phase by $1 / 4$ period, and the horizontal stresses $\sigma_{\mathrm{x}}$ reach significantly lower values than the vertical stress $\sigma_{z}$.

Figure 3 shows how to extract force and stress data ranges to create correlations. The stress and force diagrams presented in this figure are shown in a scale compatible with absolute values, i.e. for $\mathrm{kPa}$ and $\mathrm{kN}$. The maximum stress values reaching approx. $500 \mathrm{kPa}$, and the forces on the wheel approx. $20 \mathrm{kN}$, therefore there is a large difference in the diagrams. 

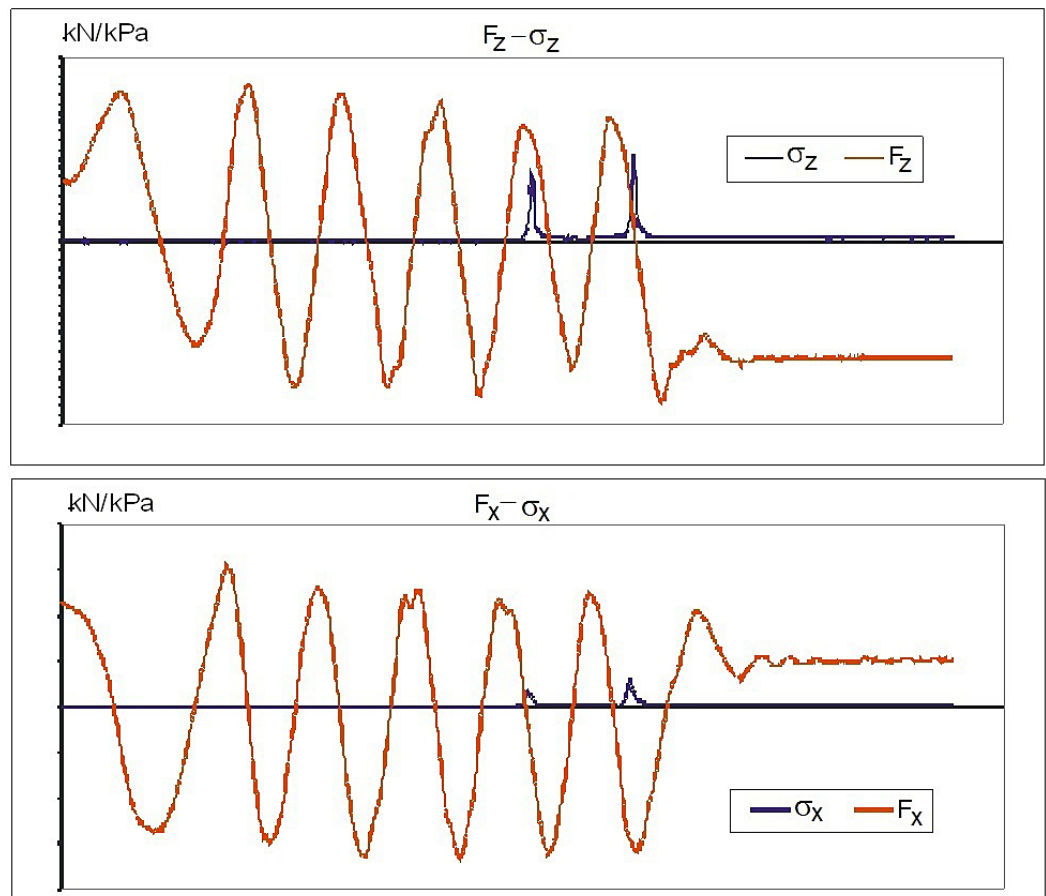

Fig. 2. Sample waveforms of forces and stresses for creating correlations
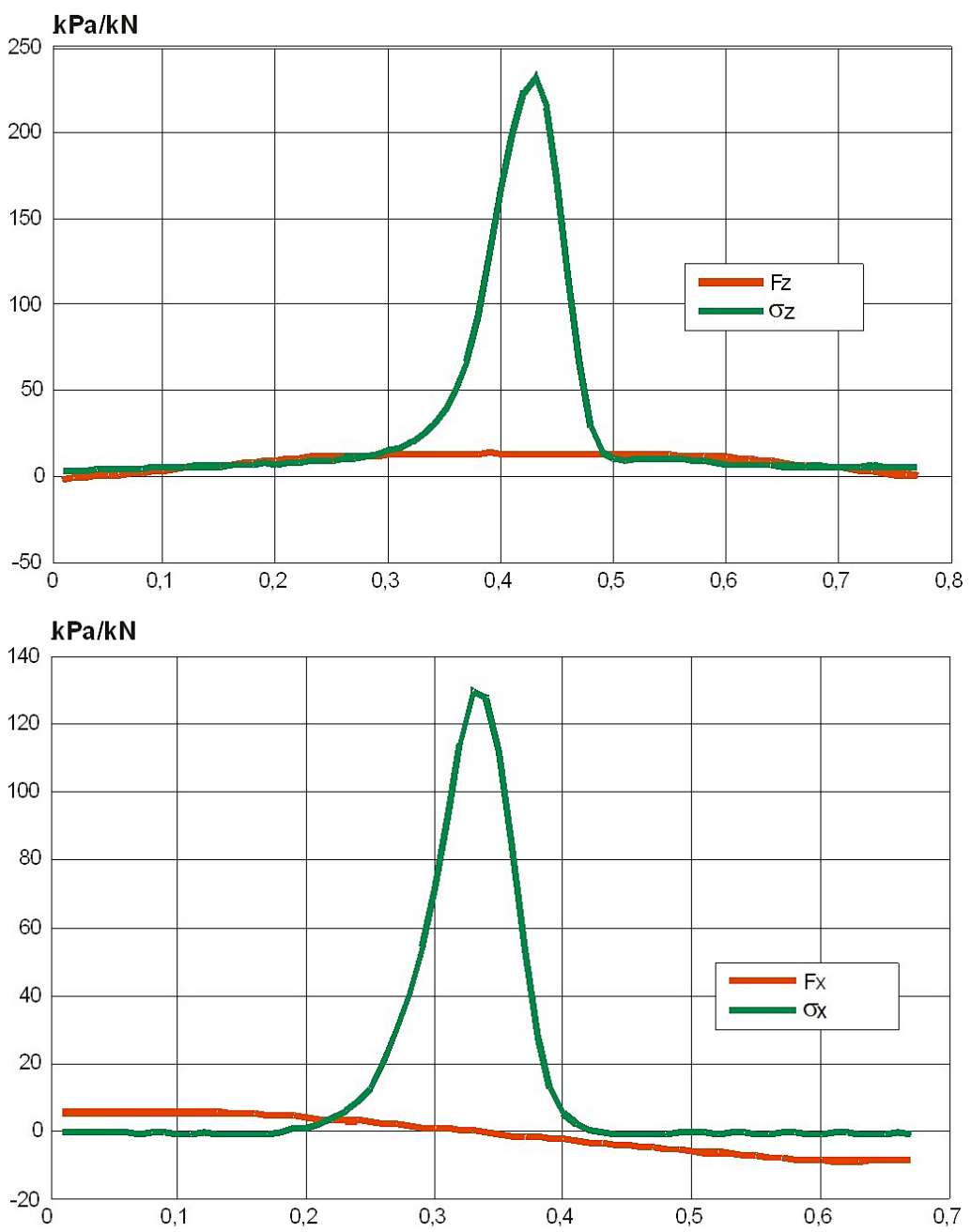

Fig. 3. Separate waveforms of forces and stresses for correlation 
Due to the fact that the results obtained in the tests with the use of the wheel force sensor are cyclometric functions and time functions, it is necessary to take this into account in the elaboration of the results and preparation of the separated data ranges for the creation of correlation. The signals with cyclometric characteristics include $F_{x}, F_{z}, M_{y}$ and $M_{z}$. The other signals are functions of time, not dependent on the angular position of the wheel. As a result, in order to obtain simple waveforms (as a function of time) of the mentioned forces and moments, it is necessary to recalculate them taking into account the angular position of the wheel. On the other hand it is good, that in the case of these considerations - where there are stress waveforms recorded during the wheel travel (i.e. cyclometric waveforms) - it is advantageous to use the force waveforms directly from the sensor, without recalculation. In this way, the obtained relationships and correlations will be correct, as they will be created from cyclometric waveforms.

When analyzing the waveforms in Figure 3 it can be seen, that while for the $F_{z}-\sigma_{z}$ set the monotonicity of both functions is consistent (the values of force and stress increase until the local maximum is reached, then they decrease in phase), in the case of the $F_{x}-\sigma_{x}$ set with phase shift. It results from a 90-degree shift in the $F_{x}$ force curve in relation to $F_{z}$ (sensor construction). This results in a different nature of the correlation for $F_{x}$, which will be shown in the following figures.

The experiment was carried out in accordance with the presented principles for three types of substrate: sandy, loess and peat. The SST sensors were placed at a depth of 10 and $20 \mathrm{~cm}$. Figures 4-6 show the results in the form of correlation of the force values $F_{z}$ and $F_{x}$ with the stress state components $\sigma_{z}$ and $\sigma_{x}$, which will be discussed below.

Figure 4 shows the results for the three soils on which the measurements were made. The relation$\operatorname{ship} F_{z}$ and $F_{x}$ has the shape of a closed curve, usually in the form of a loop somewhat reminiscent of the hysteresis of a viscoelastic material. A suggestion is made to interpret the shape of the obtained dependence curves, taking into account the three-phase nature of the soil. Analyzing the shape of the obtained curves, it is stated that the viscoelastic nature of loess and peat soils caused that the graphical representations of the $F_{x}-\sigma_{x}$ relationship for these two soils confirm the above thesis: in both cases there is a significant "hysteresis". However, sandy soil did not show this property, which is logical - because the tests were carried out at its minimum moisture.
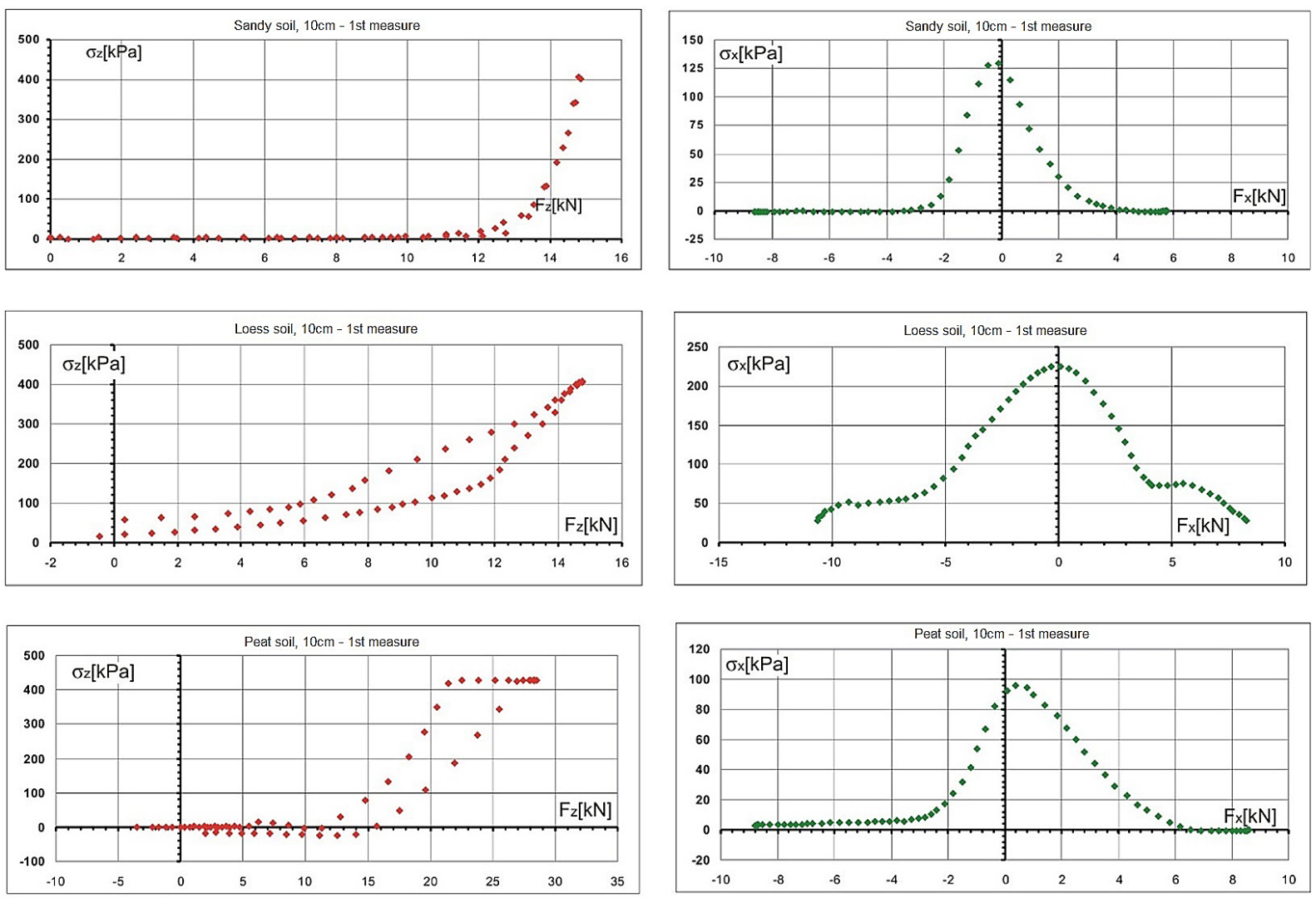

Fig. 4. Correlations of traction forces with soil stress for three different soils 

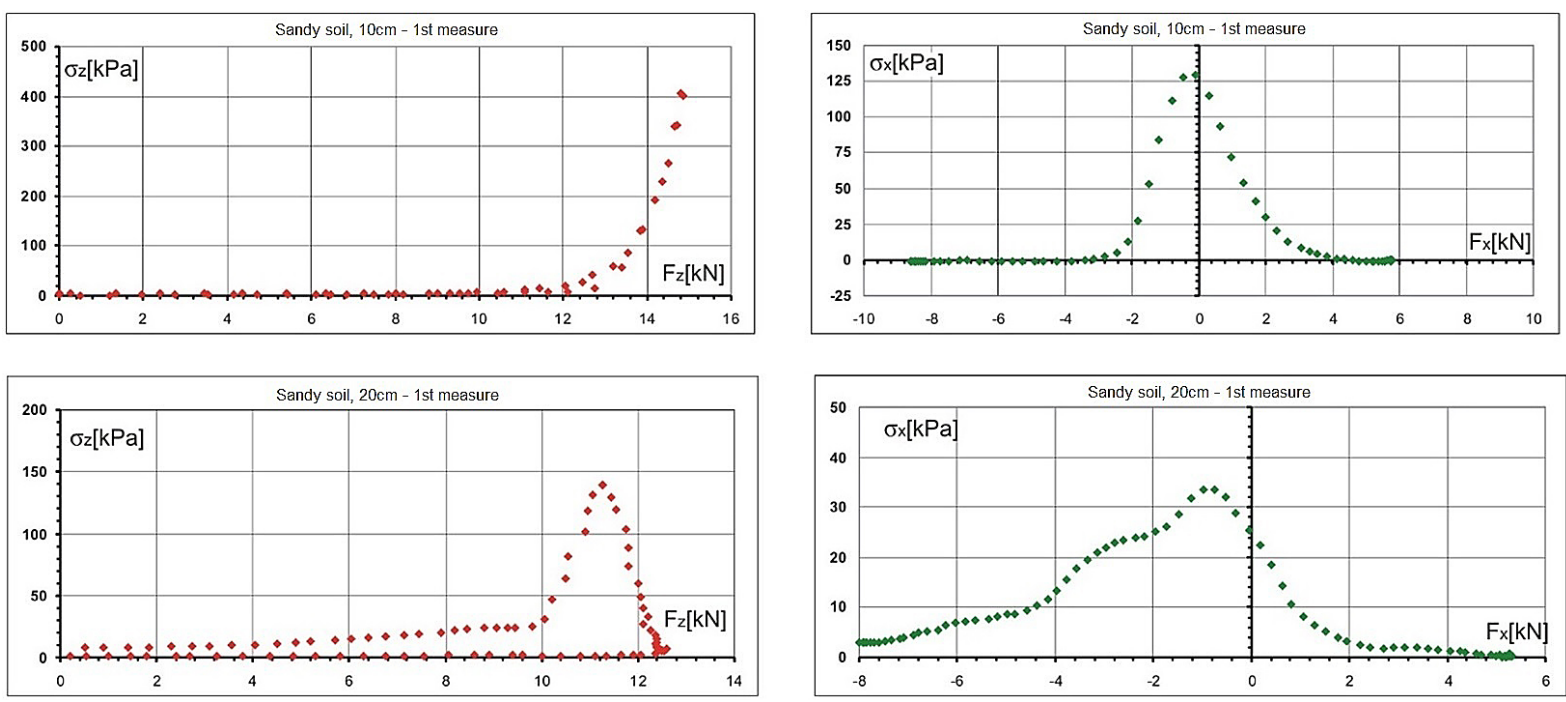

Fig. 5. Correlations of traction forces with the soil stresses for two measurement depths: 10 and $20 \mathrm{~cm}$

The share of viscosity caused by the water content between soil particles was minimal.

The $F_{x}-\sigma_{x}$ relationship has a completely different course, which results mainly from the phase shift of the components of this relationship. The so-called bell curves are received, with the maximum point at zero value of the argument of the function (force $F_{x}$ ). The obtained relationships for three different soils are similar in the case of sandy and peat soils. The third soil (loess) showed a different nature of the relationship. The difference was determined on the basis of the slope growth rate on the negative side of the graph: for sandy and peat soils it is much higher than for loess soils, moreover for these two soils the growth is almost linear. On the contrary, the loess soil shows a higher degree curve growth. Similarly, part of the curve describing soil unloading is linear or almost linear for sand and peat, while for loess it has the shape of a 3rd degree curve. Interpretation of these results on the basis of the properties of individual soils suggests the influence of consolidation and strengthening in the case of loess. However, in the case of sandy and peat soils, we are dealing with a visco-elastic reaction.

Figure 5 shows the results obtained for sandy soil. It presents the relationships $F_{z}-\sigma_{z}$ and $F_{x}-\sigma_{x}$ obtained from measurements made at two depths, 10 and $20 \mathrm{~cm}$. The differences in the shape of the obtained curves describing the relationships are significant. For the $F_{z}-\sigma_{z}$ relationship, the depth effect is visible by enlarging the hysteresis loop. This is logical, as the greater volume of the active half-space of the medium takes part in the accommodation of the load from the vehicle wheel. This results in a greater energy dissipation and a significant shift of the unloading curve in relation to the loading process. A similar trend was observed for the other two soils. As for the $F_{x}-\sigma_{x}$ relationship, the impact of the depth of the stress measurement became visible in the change of the character and width of the bellshaped curve. While for a depth of $10 \mathrm{~cm}$, the slope growth characteristic is almost linear, and the curve width is small, the measurements at a depth of 20 $\mathrm{cm}$ gave a relationship described by a much wider curve. Additionally, part of the curve describing the slope rise is not linear - but the curve of higher order. The interpretation of the results of the mechanical properties of the soil for this case also suggests the effect of a more intense dissipation of energy in the active half-space of the medium. The change in the nature of the slope rise curve can be explained by other effects, such as the consistency of deeper soil layers - where the moisture content is usually higher than just below the soil surface.

Figure 6 shows the results obtained for peat soil, obtained for five consecutive runs of the vehicle (wheel). The figure shows the relationship $F_{z}-\sigma_{z}$ and $F_{x}-\sigma_{x}$ obtained from measurements at a depth of $10 \mathrm{~cm}$. The influence of successive wheel runs is not clear. Moreover, the general nature of the relationship, described by its shape, does not change. The $F_{x}-\sigma_{x}$ relationship for individual wheel runs is described by a loop diagram, while the second of the tested relationships $\left(F_{z}-\sigma_{z}\right)$ is graphically represented by a bell curve. A detailed analysis of the shape of the $F_{z}-\sigma_{z}$ curves shows that the area of the loop decreases with successive passes. The exception to this trend is the relationship obtained for the fourth pass, when the loop area is again 

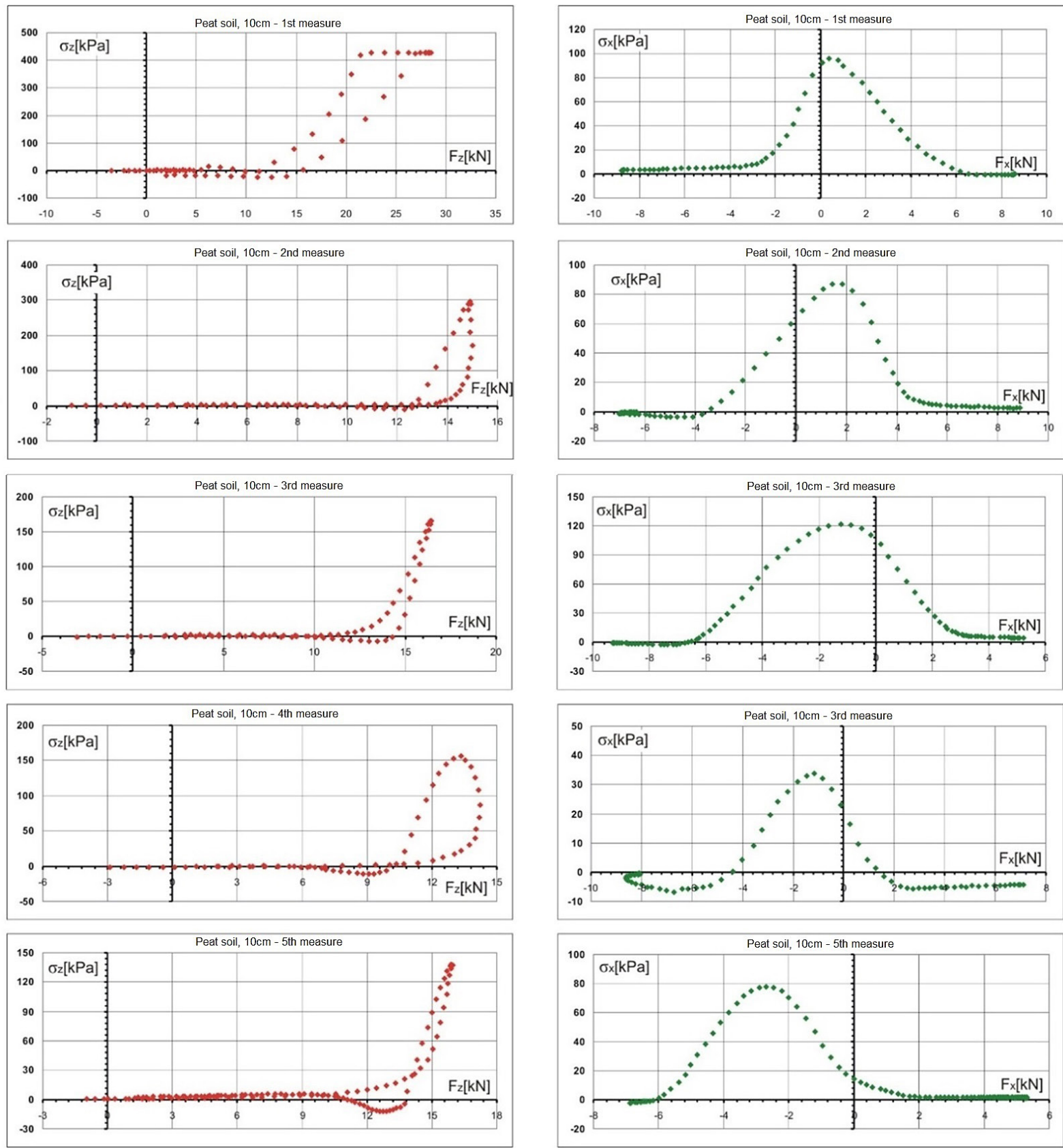

Fig. 6. Correlations of traction forces with soil stress for 5 consecutive wheel runs. Peat soil

larger, similar to the first pass. The tendency of the hysteresis loop area to decrease is logical, as each successive pass causes compaction and strengthening of the soil half-space and energy dissipation decreases as a result of soil compaction. Although the results presented in Figure 6 refer to peat soil, a similar trend was observed for all three soils - on which the research was conducted.

When analyzing the $F_{x}-\sigma_{x}$ relationship, a different peculiarity is found. The local extreme point (maximum) of the curve shifts towards negative values. During the first pass, this point was close to the stress axis - slightly positive. On the second run, it slightly shifted towards positive values. From trip
3 onwards, you can see the maximal point moving towards negative values. This means that the maximum tangential stress occurs before the wheel center reaches the measuring point. In other words, the stress "overtakes" the force it causes.

\section{CONCLUSION}

The article presents a proposal of a semi-empirical model of a wheel - soil system. It was assumed that the system in question is uniquely described by the relationships between traction forces and the components of the stress state in the soil. Measurements 
of the values characterizing this cooperation were carried out with the use of a measuring and test stand, consisting of the SST sensor for measuring the soil stress components and a dynamometer for measuring forces acting on the road wheel. The research was carried out on three different soils: sandy, loess and peat. Based on the results obtained, the following conclusions and observations can be drawn:

- the $F_{z}-\sigma_{z}$ relationship is described by the hysteresis curve, where the loop area depends on the type of soil and the measurement depth, and also changes for successive passes in one trace; this implies the conclusion that the graphical representation of the proposed model can be used in the energy analysis of the wheel-soil cooperation process,

- the $F_{x}-\sigma_{x}$ relationship is represented by a bellshaped curve, whose characteristic features - width and apex location - depend on the parameters tested (soil type, depth of the measurement point, successive track run).

The above prompts to undertake further research, the aim of which will be a strict definition of the parameters of the obtained curves, as well as the determination of their dependence on the construction and operational parameters of the vehicle, as well as on the driving dynamics.

\section{REFERENCES}

1. Komandi G. An evaluation of the concept of rolling resistance. Journal of Terramechanics. 1999;36:159-166.

2. Aubel T. FEM simulation of the interaction between elastic tyre and soft soil. $11^{\text {th }}$ Int. Conference of the ISTVS, Lake Tahoe, Nevada USA 2005, 2:791-802.

3. Bekker M.G. Introduction to terrain - vehicle system. The University of Michigan Press, Ann Arbor; 1969.

4. Muro T. Tractive performance of a driven ridig wheel on soft ground based on the analysis of soil - wheel interaction. Journal of Terramechanics. 1993;30(5):351-369.

5. Shoop S.A., Young B., Alger R., Davis J. Effect of test method on winter traction measurements. Journal of Terramechanics. 1994;31(3):153-161.

6. Wanjii S., Hiroma T., Ota Y., Kataoka T. Prediction of wheel performance by analysis of normal and tangential stress distributions under the wheel - soil interface. Journal of Terramechanics. 1997;34(3):165-186.

7. Wulfsohn D., Upadhyaya S. Prediction of traction and soil compaction using three-dimensional soil
- tyre contact profile. Journal of Terramechanics. 1992;29(6):541-564.

8. Akker van den J.J.H SOCOMO: a soil compaction model to calculate soil stresses and the subsoil carrying capacity. Soil\&Tillage research. 2004;79:113-127.

9. Bailey A.C., Raper R.L., Way T.R., Burt E.C., Johnson C.E., Soil stresses under a tractor tire at various loads and inflation pressures. Journal of Terramechanics. 1996;33(1):1-11.

10. Harris H.D., Bakker D.M. A soil stress transducer for measuring in situ soil stresses. Soil \& Tillage Research. 1994(29):35-48.

11. Bakker D.M., Harris H.D., Wong K.Y., Measurements of stress paths under agricultural vehicles and their interpretation in critical state space. Journal of Agricultural Engineering Research. 1995;61:247-260.

12. Pacejka H.B. Tire and Vehicle Dynamics. Butterworth-Heinemann, 2012.

13. Pytka J.A. Effects of steering dynamics upon tyre lateral forces on deformable surfaces. Int. Journal of Vehicle Design. 2014;64(2,3,4):170-194.

14. Pytka J.A. Identification of Rolling Resistance Coefficients for Aircraft Tires on Unsurfaced Airfields, Journal of Aircraft, AIAA Journal of Aircraft. 2014;51(2):353-360.

15. Pytka J., Józwik J., Budzyński P., Łyszczyk T., Tofil A., Gnapowski E., Laskowski J. Wheel dynamometer system for aircraft landing gear testing, Measurement. 2019;148:106918.

16. Pytka J., Budzyński P., Łyszczyk T., Józwik J., Michałowska J., Tofil A., Błażejczak D., Laskowski J. Determining Wheel Forces and Moments on Aircraft Landing Gear with a Dynamometer Sensor. Sensors. 2020;20:227. DOI: 10.3390/s20010227

17. Dąbrowski J., Pytka J., Tarkowski P., Zając M. Advantages of all-season versus snow tyres for off-road traction and soil stresses. Journal of Terramechanics. 2006;43:163-175.

18. Pytka J., Dąbrowski J., Zając M., Tarkowski P. Effects of reduced inflation pressure and vehicle loading on off-road traction and soil stress and deformation state. Journal of Terramechanics. 2006;43:469-485.

19. Pytka J. Design consideration and calibration of pressure transducer for soil stress measurements. Journal of Terramechanics. 2009;46:241-249.

20. Pytka J. Determination of snow stresses under vehicle loads. Cold Regions Science and Technology. 2010;60:137-145.

21. Pytka J., Tarkowski P., Fijałkowski S., Budzyński P., Dąbrowski J., Kupicz W., Pytka P. An Instrumented Vehicle for Off-Road Dynamics Testing. Journal of Terramechanics. 2011;48(5):384-395 